\title{
Risk Aversion and Planning Horizons
}

\author{
Antoine Bommier \\ (CNRS and Université de Toulouse, GREMAQ) \\ and \\ Jean-Charles Rochet \\ (Université de Toulouse, GREMAQ-IDEI, and Toulouse Business School)
}

First Version: August 2003

Final Version: October 25, 2005

\begin{abstract}
A number of empirical studies seem to reject the additive separability of preferences that is assumed in most theoretical models of the life cycle. We show that, when additive separability is abandoned and interactions between consumptions at different dates are taken into account, an interesting relation emerges between risk aversion and length of the planning horizon. Specifically, we show that when consumptions at different dates are specific substitutes, risk aversion increases with horizon length. This may explain the surprising empirical finding that individuals seem to increase the share of wealth held in risky assets as they become older.
\end{abstract}

(JEL: D11, D91, G11)

Acknowledgments: This paper was presented at the Third Workshop of the RTN Project on Economics of Aging, London, October 2-4, 2003, and at the Fourth Workshop of the 
RTN Project on Financing Retirement in Europe, Louvain-la-Neuve, May 14-15, 2004.

We thank the participants for their comments. We also benefited from the comments of an associate editor, Xavier Vives, and three anonymous referees. The advice of the copyeditor, Matt Darnell was also very helpful.

Email addresses: Bommier <Antoine.Bommier@univ-tlse1.fr>, Rochet <rochet@cict.fr>. 


\section{Introduction}

For many economic issues, such as for the design of Social Security or the management of pension funds, it is fundamental to know how individuals' readiness to take financial risks may change as they grow older.

Such a question has been intensively addressed in the economic literature. In the standard additive model with CRRA (constant relative risk aversion) preferences, Merton (1969) and Samuelson (1969) find that it is optimal to invest in risky assets a fraction of wealth that is independent of age. Samuelson (1989) offers a clear explanation of this result, which contradicts conventional wisdom. Later contributions try to find ways to escape this surprising result. Bodie et al. (1992) incorporate endogenous labor supply into the initial Merton-Samuelson model. They find that, if labor supply is less flexible at older ages than at younger ages, then relative risk aversion increases with age. Another important contribution is that of Gollier and Zeckhauser (2002), who show that-because of the dynamic aspect of portfolio choice problems - the conclusion of Samuelson does not extend to all additively separable preferences.

This paper contributes to the literature by examining the role of nonseparability of preferences. The assumption that preferences are additively separable has been consistently rejected by empirical evidence (see e.g. Muellbauer 1988; Carrasco, et al. 2005). Nonetheless, this assumption is still extensively made, essentially because it is so convenient. This assumption 
is, however, crucial for discussing the impact of horizon length on attitudes toward financial risks. In fact, we show that if additive separability is not assumed then individuals' relative risk aversion will generally change during the life cycle independently of any age or wealth effects and even with time consistent preferences. Moreover, we relate the life-cycle variations of relative risk aversion to standard measures of complementarity and substitutability of consumption occurring at different stages. Roughly speaking, we find that if levels of consumption at different life-cycle stages are specific substitutes then relative risk aversion indices decrease during the life cycle, whereas such indices increase if consumptions at different stages are specific complements.

Our results thus provide a simple explanation of why risk aversion may change during the life cycle: individuals' preferences may simply not be additively separable. Moreover, our results also indicate that relaxing the assumption of additive separability does not entail insuperable complexity. Even when it proves difficult or impossible to solve explicitly for intertemporal consumption-portfolio choices, it is still possible to deduce how relative risk aversion varies during the life cycle by looking at intertemporal budget shares, Frisch's cross-price elasticities, and single-period indices of risk aversion.

The remainder of this paper is organized as follows. In Section 2 we briefly review the empirical literature on the relation between risk aversion 
and age or horizon length. In Section 3 we study, as an illustrative example, a simple model of portfolio choice with nonseparable preferences. Section 4 defines a natural measure of intertemporal risk aversion and shows how this measure is related to portfolio choice. In Section 5, we consider the case of preferences that are separable but not necessarily additive in order to stress the impact of additivity on risk aversion. In Section 6 we examine the general case where both separability and additivity are relaxed. The empirical implications of our results are discussed in Section 7, and Section 8 concludes.

\section{Empirical Findings}

Before delving into theoretical considerations, we may wonder whether empirical studies suggest any relation between horizon length (or age) and risk aversion. The most direct way to assess such intertemporal risk aversion would be to elicit individuals' preferences over lotteries on their lifetime income. However, it is rather difficult to observe situations where individuals can actually choose between several lotteries on their lifetime income. This is why most empirical papers base their estimates on "virtual" experiments. For example, Barsky et al. (1997) find that the relation between relative risk aversion and age has an inverse $\mathrm{U}$ shape, although their sample is restricted to people older than 50. In contrast, Guiso and Paiella (2001) find a positive relation between risk aversion and age. However, these results should be in- 
terpreted with caution since they are based on hypothetical choices rather than actual behavior. Also, they are cross-sectional studies and therefore do not allow for control of cohort effects.

Another approach is to look at the share of wealth held in risky assets and then see how it changes during the life cycle. There are many studies that follow this track, including two recent books providing international comparisons (Guiso et al. 2002a,b) and a longitudinal study by Ameriks and Zeldes (2001). The cross-sectional studies reported in Guiso and colleagues provide mixed evidence on how the share of wealth held in risky assets varies with age. In most cases, no significant relation between age and the share of risky assets is found, although a U-shaped relation is found in the Netherlands and a weak positive relation is found in the United States. Longitudinal studies are rare. The well-documented study of Ameriks and Zeldes concludes that, after controlling for cohort effects, there is a strong positive relation in the United States between age and the share of financial portfolios held in risky assets.

It should be stressed that the empirical studies reported in Guiso et al. (2002a,b) and that of Ameriks and Zeldes (2001) analyze the share of financial wealth held in risky assets. None of them reports the share of total wealth (including human wealth) held in risky assets, which —as is made clear in Bodie et al. (1992) — would be the relevant information for assessing individual relative risk aversion. The share of total wealth held in 
risky assets is related to the share of financial wealth held in risky assets as follow:

$$
\begin{aligned}
& \frac{\text { risky assets }}{\text { human wealth }+ \text { financial wealth }} \\
& =\left(\frac{\text { risky assets }}{\text { financial wealth }}\right)\left(\frac{1}{1+(\text { human wealth }) /(\text { financial wealth })}\right) .
\end{aligned}
$$

Because the ratio of human wealth to financial wealth tends to decline with

advancing age, studies that focus on the ratio of risky assets to financial wealth tend to underestimate the (positive) slope of the relation between age and the degree of aggregate risk taking.

Thus, the absence of a relation between age and portfolio composition that is reported in cross-sectional studies - together with the positive relation between age and portfolio risk that is found in the longitudinal study of Ameriks and Zeldes - is consistent with a positive relation between age and relative risk tolerance if we take human wealth into account. Such a relation cannot be attributed to life-cycle variations in wealth, for at least two reasons. First, most studies do control for individual wealth. Second, it is generally found that relative risk aversion decreases with wealth and that wealth tends to decline toward the end of the life cycle. Hence relative risk aversion should increase with age, but the data actually support the opposite conclusion. This paper shows that nonseparability of preferences may explain this contradiction. 


\section{Portfolio Choice with Nonseparable Preferences:}

\section{An Illustrative Example}

As mentioned in the Introduction, the aim of this paper is to discuss, in a very general framework, the relationship between risk aversion and the planning horizon in order to explain why the optimal degree of risk taking may change along the life cycle. For our most general result (see Section 6) we will assume only that agents are risk averse and have preferences that can be represented within the expected utility framework. The cost for such generality is that it is then impossible to derive exact analytical solutions for standard portfolio choice problems. Our results must then rely on marginal properties of the indirect utility function and thus are only local.

In some particular cases, however, exact solutions and global results can be derived. We develop one such case in this section, since this will help us examine the link between standard portfolio choice problems and our more abstract discussion on risk aversion. The model is a discrete and simplified version of the one in Pye (1973). Individuals live two periods and have a lifetime von Neumann-Morgenstern utility function given by

$$
U\left(C_{1}, C_{2}\right)=f\left(u\left(C_{1}\right)+u\left(C_{2}\right)\right)
$$

where $u(C)=\ln C$ is the instantaneous utility function ${ }^{1}$ and $f(A)=1-$

\footnotetext{
${ }^{1}$ To neutralize age effects, we assume that the instantaneous utility is independent of
} age. 
$e^{-k A / k}$

The coefficient $k$ measures interactions between consumptions on different dates:

$$
\frac{\partial^{2} U}{\partial C_{1} \partial C_{2}}\left(C_{1}, C_{2}\right)=-\frac{k}{C_{1} C_{2}} e^{-k\left(u\left(C_{1}\right)+u\left(C_{2}\right)\right)} .
$$

Thus, consumptions at dates 1 and 2 are "want independent" (additively separable preferences) $)^{2}$ if $k=0$, specific substitutes if $k>0$, and specific complements if $k<0$. See Section 6 for the precise definitions of these terms.

At date 0 , the individual invests a fraction $\theta_{0}$ of her initial wealth $W_{0}$ in a risky asset, with the rest being invested in a riskless asset. At date 1 she has wealth $W_{1}$, out of which she chooses to consume $C_{1}$, so her remaining wealth is now $W_{1}-C_{1}$. She then invests a fraction $\theta_{1}$ of her remaining wealth in the same risky asset. At date 2 , she consumes her final wealth $W_{2}$. We denote by $\tilde{R}_{0}$ and $\tilde{R}_{1}$ the (random) returns of the risky asset in periods 0 and 1, returns that are presumed to be i.i.d. The riskless return is normalized to zero, and we assume that the risky asset has a positive expected return of $R$. The budget constraints are given by

$$
\begin{aligned}
W_{1} & =W_{0}\left(1+\theta_{0} \tilde{R}_{0}\right), \\
C_{2} & =\left(W_{1}-C_{1}\right)\left(1+\theta_{1} \tilde{R}_{1}\right) .
\end{aligned}
$$

We focus on the evolution of the share of risky assets during the life cycle - that is, on whether or not $\theta_{0}>\theta_{1}$.

\footnotetext{
${ }^{2}$ When $k=0$, we adopt the usual convention that $f(A)=A$.
} 
Observe first that, in the additively separable case $(k=0)$, this share is constant: $\theta_{0}=\theta_{1}$. Indeed, at date 1 the individual chooses $\theta_{1}$ to maximize $E\left[\ln \left(1+\theta_{1} \tilde{R}_{1}\right)\right]$. The share $\theta_{1}$ does not depend on $W_{1}-C_{1}$, because $u(C)=$ $\ln C$ is CRRA. Consumption $C_{1}$ is chosen to maximize $\ln C_{1}+\ln \left(W_{1}-C_{1}\right)$, which leads to

$$
C_{1}=W_{1}-C_{1}=\frac{1}{2} W_{0}\left(1+\theta_{0} \tilde{R}_{0}\right)
$$

By backward induction, $\theta_{0}$ is chosen at date 0 in order to maximize

$$
E\left[\ln C_{1}+\ln \left(W_{1}-C_{1}\right)\right]=\text { constant }+2 E\left[\ln \left(1+\theta_{0} \tilde{R}_{0}\right)\right]
$$

As a result, if $\tilde{R}_{0}$ and $\tilde{R}_{1}$ are identically distributed then $\theta_{0}$ and $\theta_{1}$ coincide: the share of risky assets in the portfolio of the individual is constant across the life cycle. Of course, this is due in part to the fact that we have neutralized age effects and wealth effects. We claim that this is due also to intertemporal additive separability.

In order to see this, consider now the nonadditive case $(k \neq 0)$ :

$$
U\left(C_{1}, C_{2}\right)=\frac{1-e^{-k\left(\ln C_{1}+\ln C_{2}\right)}}{k}=\frac{1-C_{1}^{-k} C_{2}^{-k}}{k} .
$$

At date 1 , the individual chooses $\theta_{1}$ to maximize $E\left[U\left(C_{1},\left(W_{1}-C_{1}\right)(1+\right.\right.$ $\left.\left.\left.\theta_{1} \tilde{R}_{1}\right)\right)\right]$. Again, the optimal $\theta_{1}$ is independent of $C_{1}$ and $W_{1}$ because instantaneous utility is CRRA (no wealth effect):

$$
\theta_{1}=\arg \max \left[-E\left(1+\theta_{1} \tilde{R}_{1}\right)^{-k}\right]
$$

As in the additively separable case, $C_{1}$ is chosen to maximize $\ln C_{1}+$ $\ln \left(W_{1}-C_{1}\right)$, leading to $C_{1}=W_{1} / 2$, but the choice of $\theta_{0}$ is changed. The 
objective function becomes $-E\left[C_{1}^{-k} C_{2}^{-k}\right]$, which is proportional to

$$
-E\left[\left(1+\theta_{0} \tilde{R}_{0}\right)^{-2 k}\right]
$$

We thus obtain an expression similar to (3) but with a different exponent. This is because the risk on the portfolio chosen at $t=0$ affects two consumption levels, $C_{1}$ and $C_{2}$, whereas $\theta_{1}$ affects only $C_{2}$. When preferences are not additively separable, this changes the portfolio decision.

Specifically, in our example it is easy to see that $\theta_{0}<\theta_{1}$, when $k>0$, which means that the individual takes more risk at date 1 . Indeed, this is an easy consequence of the following comparative statics property (proved in the Appendix).

Lemma 1. Let

$$
\theta^{*}(k)=\arg \max \left\{\frac{1-E\left[(1+\theta \tilde{R})^{-k}\right]}{k}\right\}
$$

Then $\theta^{*}$ decreases in $k$.

If $R$, the expected return on the risky asset, tends to zero while its variance $\sigma^{2}$ remains constant, then we can derive a simple approximation of $\theta_{0}$ and $\theta_{1}$. A second-order Taylor expansion of (3) shows that, when $R \rightarrow 0$,

$$
\theta_{0} \approx \frac{1}{1+2 k} \frac{R}{\sigma^{2}} \quad \text { and } \quad \theta_{1} \approx \frac{1}{1+k} \frac{R}{\sigma^{2}}
$$

As we shall see, this approximation could have been obtained from considerations of risk aversion alone and without solving the portfolio choice 
problem: the coefficients that appear before $R / \sigma^{2}$ in $(5)$ are the intertemporal risk tolerance indices of the individual, as defined in the next section.

\section{Intertemporal Risk Aversion}

The example of Section 3 shows that relaxing the assumption of additive separability may significantly affect the relation between age and financial strategies. But so far, it is difficult to tell what drives the result. Is it a peculiarity of our simple model of portfolio choice, or does it reflect a fundamental aspect of nonadditive preferences? The latter hypothesis is actually the correct one. As we explain in what follows, interactions between levels of consumption at different dates are a key determinant of the relation between intertemporal risk aversion and horizon length. Moreover, since financial strategies are closely related to intertemporal risk aversion, we also find that portfolio choice depends on horizon length when preferences are not additively separable. Unfortunately, optimal portfolio selection with nonseparable preferences is a formidable computational problem, with no hope for a closed-form solution except in special cases. So our strategy will be (i) to define an intertemporal measure of risk aversion in a neighborhood of a deterministic consumption profile (the risk tolerance index at age $n$ ), and (ii) to study how this index varies during the life cycle. Then we will show that this index allows us to obtain a good approximation of the share

of risky assets in the portfolios chosen by individuals of different ages — at 
least when the excess return of the risky asset is small.

Before introducing theoretical considerations on intertemporal risk aversion, a natural question arises: How can we compare risk aversions of individuals who have different planning horizons? Individuals with different horizons do indeed have preferences over different consumption sets. Comparative risk aversion was originally developed by Arrow (1971) and Pratt (1964) for preferences over a single commodity. It was extended by Kihlstrom and Mirman (1974) to the case where people consume several goods but have the same ordinal preferences. Clearly, this cannot be applied to individuals who consume over different numbers of periods. Karni $(1979,1983)$ suggested alternative approaches to multivariate comparative risk aversion; once again, however, no clear comparison can be obtained when applying these approaches to individuals who care for different goods. Thus, strictly speaking there is no theoretical foundation for comparing risk aversions of individuals with different horizon lengths.

Yet it may be possible to compare the degree of risk aversion individuals' indirect utility functions. Again, there are various options. The comparison can be in terms of relative or absolute risk aversion. Also, since individuals of different ages may possess different amounts of wealth, it is not clear whether we should control for wealth variations or not. We will define an intertemporal index of risk tolerance and explain it as a natural measure for analyzing how an individual's attitude toward risk varies during the life 
cycle.

Consider then an individual with a lifetime utility function $U\left(C_{1}, C_{2}, \ldots, C_{N}\right)$, and assume that these utilities are "time consistent". More formally, this means that an agent of age $n$ with past consumption $\left(C_{1}^{*}, C_{2}^{*}, \ldots, C_{n-1}^{*}\right)$ has preferences over $\left(C_{n}, \ldots, C_{N}\right)$ that are represented by the utility function

$$
U_{n}\left(C_{n}, \ldots, C_{N}\right)=U\left(C_{1}^{*}, C_{2}^{*}, \ldots, C_{n-1}^{*}, C_{n}, \ldots, C_{N}\right)
$$

The price of the composite good consumed in period $i$ is denoted $p_{i}$. In the absence of uncertainty, individuals with initial wealth $W$ initially choose the consumption path $\left(C_{1}^{*}, C_{2}^{*}, \ldots, C_{N}^{*}\right)$ that maximizes $U\left(C_{1}, \ldots, C_{N}\right)$ under the budget constraint $\sum_{i=1}^{N} p_{i} C_{i}=W$. At any age $n$, the remaining wealth is $W_{n}=W-\sum_{i=1}^{n-1} p_{i} C_{i}^{*}$ and individuals then choose $\left(C_{n}, \ldots, C_{N}\right)$ so as to maximize $U_{n}\left(C_{n}, \ldots, C_{N}\right)$ under the budget constraint $\sum_{i=n}^{N} p_{i} C_{i}=$ $W_{n}$. The time consistency assumption implies that the solution to the maximization program at age $n$ is given by $\left(C_{n}^{*}, \ldots, C_{N}^{*}\right)$ and hence that individuals stick to their initial choices.

Definition 1. The intertemporal risk tolerance index at age $n$ along consumption path $C^{*}=\left(C_{1}^{*}, \ldots, C_{N}^{*}\right)$ is defined as

$$
T_{n}\left(C_{1}^{*}, \ldots, C_{N}^{*}\right)=-\frac{V_{n}^{\prime}\left(W_{n}\right)}{W_{n} V_{n}^{\prime \prime}\left(W_{n}\right)},
$$

where $W_{n}=W-\sum_{i=1}^{n-1} p_{i} C_{i}^{*}$ is the wealth held at age $n$ and $V_{n}(\cdot)$ is the 
value function of an individual of age $n$ with utility function $U$ :

$$
\left(\mathcal{P}_{n}\right)\left\{\begin{array}{l}
V_{n}\left(W_{n}\right)=\max _{C_{n}, \ldots, C_{N}}\left\{U\left(C_{1}^{*}, \ldots, C_{n-1}^{*}, C_{n}, \ldots, C_{N}\right)\right\}, \\
\sum_{i=n}^{N} p_{i} C_{i}=W_{n} .
\end{array}\right.
$$

To illustrate why this index of risk tolerance is informative about how attitudes toward risk change during the life cycle, we now consider two cases in which some marginal uncertainty is added to the deterministic setting already described.

For the first illustrative case, imagine that at age $n$ the individual is offered a choice between giving up a share $\alpha_{n}$ of his wealth (leaving him with wealth $\left.\left(1-\alpha_{n}\right) W_{n}\right)$ and participating in a fair lottery that provides him with wealth $(1+\varepsilon) W_{n}$ or $(1-\varepsilon) W_{n}$ with equal probability. Now ask: What is the share $\alpha_{n}(\varepsilon)$ that leaves the individual indifferent between the two alternatives? This is similar to computing a risk premium in one-dimensional analysis. It is easy to show that

$$
\alpha_{n}(\varepsilon)=\frac{\varepsilon^{2}}{2 T_{n}\left(C^{*}\right)}+o\left(\varepsilon^{2}\right)
$$

This formula means that, as a first approximation, the relative risk premium for a lottery on the individual's wealth at age $n$ is inversely proportional to the intertemporal tolerance index at age $n$, in conformity with the classical analysis of Arrow and Pratt.

Our second case consists of introducing a risky asset into the economy and then looking at the limit behavior of portfolio choices at different ages 
when the return on the risky asset tends to zero, so that the fraction of wealth held in risky assets is small. We thus extend our model of Section 3 to the $N$-period case. The return of the riskless asset is still assumed to be zero, but there is no loss of generality here since we assume that consumption at different dates may have different prices. Individuals have an initial wealth $W_{0}$. At date 0 individuals choose $\theta_{0}$, the fraction of $W_{0}$ that is invested in the risky asset. The return on the risky asset is $R_{0}$, which provides them, at date 1 , with wealth $W_{1}=W_{0}\left(1+\theta_{0} R_{0}\right)$. Then individuals choose $C_{1}$, and the fraction $\theta_{1}$ of their remaining wealth $W_{1}-p_{1} C_{1}$, invested in the risky asset. The return on the risky asset is $R_{1}$, which provides them at date 2 with a wealth $W_{2}=\left(W_{1}-p_{1} C_{1}\right)\left(1+\theta_{1} R_{1}\right)$. The consumption $C_{2}$ is chosen, and so on until period $N$, when individuals end up consuming all their wealth.

Assume that the risky returns $R_{i}$ are i.i.d. with $E\left[R_{i}\right]=R$ and $\operatorname{Var}\left(\mathrm{R}_{\mathrm{i}}\right)=$ $\sigma^{2}$. Denote by $C^{*}$ the consumption path that is chosen when there is no risky asset (or when $R=0$ ).

Lemma 2. When $R$ is close to zero, the share of wealth invested in the risky asset at date $n$ is given by

$$
\theta_{n}(R)=\frac{R}{\sigma^{2}} T_{n+1}\left(C^{*}\right)+o(R)
$$

where $o(R)$ is a function such that $\lim _{R \rightarrow 0} o(R) / R=0$.

Proof. We use $V_{n}\left(C_{1}^{*}, \ldots, C_{n-1}^{*}, W_{n}\right)$ to denote the indirect utility function 
at age $n$. By definition

$V_{n}\left(C_{1}^{*}, \ldots, C_{n-1}^{*}, W_{n}\right)=\left\{\max _{\theta_{n}, C_{n}} E\left[V_{n+1}\left(C_{1}^{*}, \ldots, C_{n},\left(W_{n}-p_{n} C_{n}\right)\left(1+\theta_{n} R_{n}\right)\right)\right]\right\}$

The first-order condition of this problem with respect to $\theta_{n}$ gives

$$
E\left[\left(W_{n}-p_{n} C_{n}\right) R_{n} V_{n+1}^{\prime}\left(C_{1}^{*}, \ldots, C_{n},\left(W_{n}-C_{n}\right)\left(1+\theta_{n} R_{n}\right)\right)\right]=0
$$

where the derivative is taken with respect to the last argument. If $E\left[R_{n}\right]$ and thus $\theta_{n}$ is small then, after simplifying by $\left(W_{n}-p_{n} C_{n}\right)$, a Taylor expansion yields

$$
E\left[R_{n}\right] V_{n+1}^{\prime}\left(W_{n}-p_{n} C_{n}\right)+\theta_{n} E\left[R_{n}^{2}\right]\left(W_{n}-p_{n} C_{n}\right) V_{n+1}^{\prime \prime}\left(W_{n}-p_{n} C_{n}\right) \approx 0
$$

Because $E\left[R_{n}\right]=R$ is small, we can replace $E\left[R_{n}^{2}\right]$ by $\sigma^{2}$ and so obtain the desired result.

The share of wealth invested in the risky asset at date $n$ is therefore proportional, as a first-order approximation, to the intertemporal risk tolerance index $T_{n+1}\left(C^{*}\right)$. We next study how $T_{n}\left(C^{*}\right)$ changes with $n$; this will give us a first approximation of how an individual's optimal financial strategy varies during the life cycle.

\section{Risk Aversion with Separable but Not Neces- sarily Additive Preferences}

To stress the role played by the additivity assumption that is made in most studies, we consider in this section the simplest extension of the additively 
separable model. The (ordinal) assumption of separability of preferences is maintained, ${ }^{3}$ but we do not assume that the von Neumann-Morgenstern utility function is additive. From Gorman (1968) we know that ordinal separability implies that the lifetime von Neumann-Morgenstern utility function is of the form

$$
U\left(C_{1}, \ldots, C_{N}\right)=f\left(\sum_{i=1}^{N} u_{i}\left(C_{i}\right)\right) .
$$

The function $f(\cdot)$ and the instantaneous utility functions $u_{i}(\cdot)$ are assumed to be twice continuously differentiable and to have positive first-order derivatives. The shape of $f$ captures the interactions between consumption at different dates: nil if $f$ is linear, complementarities if $f$ is convex, substitutabilities if $f$ is concave.

Proposition 1. In the additively separable case (i.e., when $f$ is linear), the intertemporal risk tolerance index at date $n$ along the consumption path $C^{*}=\left(C_{1}^{*}, \ldots, C_{N}^{*}\right)$ is a weighted sum of instantaneous risk tolerance indices:

$$
T_{n}\left(C^{*}\right)=\sum_{i=n}^{N} \alpha_{i}^{n} t_{i}\left(C_{i}^{*}\right)
$$

where $\alpha_{i}^{n}=p_{i} C_{i}^{*} /\left(\sum_{j=n}^{N} p_{j} C_{j}^{*}\right)$ is the share of (remaining) intertemporal budget spent at date $i$ and $t_{i}\left(C_{i}^{*}\right)=-u_{i}^{\prime}\left(C_{i}^{*}\right) / C_{i}^{*} u_{i}^{\prime \prime}\left(C_{i}^{*}\right)$ is the instantaneous index of relative risk tolerance at date $i$.

\footnotetext{
${ }^{3}$ This means that the indifference curves between consumption during two different periods do not depend on consumption during other periods.
} 
This is a standard result. We do not provide a proof here because this result is a particular case of Proposition 3, which is stated in Section 6 and proven in the Appendix.

Equation (7) already shows different reasons why the risk tolerance index may vary during the life cycle. It may be that the functions $t_{i}(\cdot)$, which measure instantaneous risk tolerance, change with age; we would then have "age effects". This would be the case, for example, if (say, for some psychological reasons) older individuals prove to be more or less risk averse than younger ones with respect to instantaneous consumption. Another possibility is that the functions $t_{i}(\cdot)$ are all identical (no age effects) but that they are not constant in $C^{*}$ and that consumption changes during the life cycle; then we would have "wealth effects". However, since the weights $\alpha_{i}^{n}$ in (7) sum to unity, it is clear that - except for these age and wealth effectsthere is no other element that could cause risk aversion to change during the life cycle: if $t_{i}\left(C_{i}^{*}\right)$ is independent of $i$, then $T_{n}\left(C^{*}\right)$ is independent of $n$ and there are no horizon effects.

If instantaneous utility functions are all identical (no age effect) and CRRA (so that there is no wealth effect), then relative risk tolerance is constant over the life cycle. This explains why there is no relation between horizon length and relative risk aversion in the model of Merton (1969) and Samuelson (1969). 
From now on, we consider the effect on risk tolerance of relaxing additive separability. To do this, we neutralize age effects by assuming that, up to a time preference factor, the instantaneous utility functions are identical across dates, $\left(u_{i} \equiv \delta_{i} u\right.$ with $\left.\delta_{i}>0\right)$. We also neutralize wealth effects by considering stationary consumption paths. Proposition 2 shows that, when $f$ is nonlinear, risk tolerance indices vary during the life cycle.

Proposition 2. Along any stationary consumption path $\left(C^{*}, C^{*}, \ldots, C^{*}\right)$, the sequence of intertemporal risk tolerance indices $T_{1}, \ldots, T_{N}$ is increasing if $f^{\prime \prime}<0$, decreasing if $f^{\prime \prime}>0$, and constant if $f^{\prime \prime} \equiv 0$.

Proof. In order to sustain a stationary consumption path, prices must be proportional to $\delta_{i}$; we normalize them so that $\sum_{i \geq n} p_{i}=1$. Then $W=C^{*}$, and $V_{n}$ is explicit:

$$
V_{n}(W)=f\left[\sum_{i<n} \delta_{i} u\left(C^{*}\right)+\left(\sum_{i \geq n} \delta_{i}\right) u(W)\right] .
$$

Thus, we can immediately find $V_{n}^{\prime}$ as follows:

$$
V_{n}^{\prime}(W)=f^{\prime}(A)\left(\sum_{i \geq n} \delta_{i}\right) u^{\prime}(W)
$$

where $A$ denotes the term within brackets in (8), as computed at the stationary consumption path $(W, W, \ldots, W)$ (notice that $A$ is independent of n). Similarly, we have

$$
V_{n}^{\prime \prime}(W)=f^{\prime}(A)\left(\sum_{i \geq n} \delta_{i}\right) u^{\prime \prime}(W)+f^{\prime \prime}(A)\left(\sum_{i \geq n} \delta_{i}\right)^{2} u^{\prime 2}(W) .
$$


Therefore,

$$
R_{n}=\frac{1}{T_{n}}=-\frac{C^{*} u^{\prime \prime}\left(C^{*}\right)}{u^{\prime}\left(C^{*}\right)}-C^{*} \frac{f^{\prime \prime}(A)}{f^{\prime}(A)}\left(\sum_{i \geq n} \delta_{i}\right) u^{\prime}\left(C^{*}\right) .
$$

When $f$ is linear $\left(f^{\prime \prime}=0\right)$, risk aversion is constant along any stationary con-

sumption path $\left(C^{*}, C^{*}, \ldots, C^{*}\right)$ and is equal to the static risk aversion index $-C^{*}\left(u^{\prime \prime} / u^{\prime}\right)\left(C^{*}\right)$. However, if $f^{\prime \prime} \neq 0$ then there is a correcting term, which is positive and decreasing in $n$ when $f^{\prime \prime}<0$ but negative and increasing in $n$ when $f^{\prime \prime}>0$.

It remains to extend this analysis to the case where preferences are neither additive nor separable. This is our task in the next section.

\section{The Impact of Consumption Interactions on Risk Aversion}

The previous section made it clear that relaxing the assumption of additive separability may lead one to revise significantly the relation between horizon length and risk aversion. It would, however, be excessively optimistic to say that empirical studies have so far clearly established how consumption at different moments in time interacts with consumer preferences. Most papers that challenge the additivity assumption have proposed particular extensions of the additively separable model and then tested whether such extensions fit the data better. For example, this is the case with papers on "habit formation", which extend the standard additive model by allowing 
the marginal utility of current consumption to depend on past consumption (see e.g. Muellbauer 1988; Dynan 2000). However, the choice of these extensions is guided by intuitive arguments or by technical reasons, not by empirical evidence. The additive model is probably unrealistic, but equally doubtful is the validity of these simple extensions and of the separable but nonadditive model examined in Section 5.

Given these doubts, we consider it important to derive results that do not rely on any particular specification. In the following we therefore study the general case in which preferences are represented by a concave, and twice continuously differentiable von Neumann-Morgenstern utility function,

$$
U(C)=U\left(C_{1}, C_{2}, \ldots, C_{N}\right)
$$

without making any further assumptions. With this general formulation, we must resort to the fundamental concepts of utility theory in order to describe individual preferences. Since we are interested in the cardinal properties of the utility function, we will naturally refer to the seminal contributions of Frisch (1959) and Houthakker (1960) and will use their terminology.

Definition 2. Consumptions at dates $i$ and $j$ are specific substitutes if and only if $\left[D^{2} U\right]_{i j}^{-1}>0$. They are specific complements if and only if $\left[D^{2} U\right]_{i j}^{-1}<0$, and they are want independent if and only if $\left[D^{2} U\right]_{i j}^{-1}=0$.

With additively separable preferences, all consumptions at different periods are "want independent" because $\left[D^{2} U\right]$ (and thus $\left.\left[D^{2} U\right]^{-1}\right)$ is diagonal (or block diagonal if several goods are consumed during each period). 
Definition 3. The coefficient of specific substitutability between consumptions at dates $i$ and $j$ (for a consumption profile $C$ ) is given by

$$
\kappa_{i j}(C)=\frac{u_{i} u_{j}\left[D^{2} U\right]_{i j}^{-1}}{C_{i} u_{i}+C_{j} u_{j}}
$$

where $u_{i}=\partial U / \partial C_{i}$ for $i=(1, \ldots, N)$.

This coefficient is positive if consumptions at dates $i$ and $j$ are specific substitutes and is negative if they are specific complements. It is related to the notion (see Frisch 1959) of "want elasticity" of consumption at date $i$ with respect to consumption at date $j$ :

$$
x_{i j} \equiv \frac{u_{j}}{C_{i}}\left[D^{2} U\right]_{i j}^{-1} \quad \text { for } i \neq j .
$$

However, we prefer to use the $\kappa_{i j}$ rather than the $x_{i j}$, because the former more clearly show the symmetry of our results (the $\kappa_{i j}$ are symmetric whereas the $x_{i j}$ are not).

The following result gives a general formula linking risk tolerance indices during the life cycle to coefficients of specific substitutability between consumption at different dates (the $\kappa_{i j}$, as defined in (10)). Our formula is valid when interactions are small - that is, when $\kappa \equiv \max _{i \neq j}\left|\kappa_{i j}\right|$ is small.

Proposition 3. When interactions between consumption at different dates are small, the intertemporal risk tolerance index at age $n$ (along consumption path $C^{*}$ ) can be approximated by a weighted sum of instantaneous risk tolerance indices plus a correcting term. This correcting term is negative when 
consumptions at different dates are specific substitutes. More specifically, the relative risk tolerance at age $n$ is given by

$$
T_{n}\left(C^{*}\right)=\sum_{i \geq n} \alpha_{i}^{n} t_{i}\left(C^{*}\right)-\sum_{\substack{i, j \geq n \\ i \neq j}}\left(\alpha_{i}^{n}+\alpha_{j}^{n}\right) \kappa_{i j}+\kappa o(\kappa),
$$

where

$$
t_{i}\left(C^{*}\right)=-\frac{\frac{\partial U}{\partial C_{i}}\left(C^{*}\right)}{C_{i}^{*} \frac{\partial^{2} U}{\partial C_{i}^{2}}\left(C^{*}\right)}
$$

is the instantaneous risk tolerance index at date $i$,

$$
\alpha_{i}^{n}=\frac{C_{i}^{*} \frac{\partial U}{\partial C_{i}}\left(C^{*}\right)}{\sum_{j \geq n} C_{j}^{*} \frac{\partial U}{\partial C_{j}}\left(C^{*}\right)}
$$

is the budget share spent at date $i$ (relative to the budget to be spent in the remaining periods of life), $\kappa=\max _{i \neq j}\left|\kappa_{i j}\right|$, and $o(\kappa) / \kappa \rightarrow 0$ when $\kappa \rightarrow 0$.

Proposition 3 (see Appendix for proof) allows one to measure the bias introduced by neglecting intertemporal interactions. If $U$ is additively separable (i.e., if $U(C)=\sum_{i} u_{i}\left(C_{i}\right)$ ), then all the $\kappa_{i j}$ are zero and the intertemporal risk tolerance index $T_{n}\left(C^{*}\right)$ reduces to a weighted sum of instantaneous indices, as stated in Proposition 1. However, if consumptions at different dates are specific substitutes $\left(\kappa_{i j}>0\right)$ but such interactions remain small ( $\kappa$ small), then the relative risk tolerance at age $n$ is decreased by a factor that roughly equals a weighted sum of coefficients of specific substitutability between consumption at different dates. The adjustment for risk tolerance is therefore negative when consumptions at different dates are specific substitutes and positive when such consumptions are specific complements. More 
generally: if some goods are specific complements to themselves (at other dates) but others are specific substitutes, then the sign of the bias is given by the sum of these coefficients weighted by the budget shares.

Equation (11) suggests that, barring additive separability, there may be a gap between relative risk tolerance with respect to instantaneous consumption and relative risk tolerance with respect to wealth. If consumptions at different periods of time are specific complements, then agents may be highly risk averse with respect to instantaneous consumption and moderately risk averse with respect to wealth. This is what happens with the specification chosen by Constantinides (1990) to solve the equity premium puzzle. On the other hand, if consumptions at different dates are specific substitutes, then relative risk aversion with respect to wealth may be much larger than relative risk aversion with respect to instantaneous consumption. This is the case in the model proposed by Ahn (1989) for solving the equity premium puzzle. It is troubling, however, to see that both specific complementarity and specific substitutability were used to solve the same empirical puzzle.

The key to understanding the relationship between risk aversion and planning horizons, is to notice that the size of the correcting term that accounts for the nonseparability of preferences varies with horizon length. Indeed, this term, which is given by

$$
-\sum_{\substack{i, j \geq n \\ i \neq j}}\left(\alpha_{i}^{n}+\alpha_{j}^{n}\right) \kappa_{i j}
$$


is a sum restricted to indices $i$ and $j$ that are equal to or greater than the current age $n$. There are $(N-n)(N-n+1) / 2$ terms in that sum. However, the relative budget shares $\alpha_{i}^{n}$ are (on average) lower when the horizon length is large, since $\sum_{i \geq n} \alpha_{i}^{n}=1$ by definition. Roughly speaking (i.e., omitting the variations in the $\kappa_{i j}$ and in the $\left.\alpha_{i}^{n}\right)$, there are $(N-n)(N-n+1) / 2$ terms of size $2 \kappa /(N-n+1)$ in the sum shown in (12), which gives a term of size $\kappa(N-n)$. Hence, the correcting term increases (in absolute value) with the strength of the interaction between consumption at different dates and with horizon length. The bias due to the assumption of additive separability is therefore typically larger for younger individuals — who still have many periods to live - than for older individuals.

The reason why complementarity or substitutability of consumption at different dates affects intertemporal risk tolerance is rather intuitive. For individuals who smooth their consumption during the life cycle, a negative shock to wealth at date $n$ will yield negative shocks to consumption in all remaining periods of life; likewise, a positive shock to wealth will generate positive shocks to consumption. The point we stress is that, regardless of whether the shock to wealth is positive or negative, it generates a sequence of shocks on instantaneous consumption that are positively correlated. Risk aversion with respect to wealth is therefore akin to risk aversion with respect to positively correlated risks on instantaneous consumption. If consumptions at different periods are neither complements nor substitutes (as in the 
additive model), then it does not matter that the risks on instantaneous consumption are positively correlated. However, as soon as there are substitutabilities or complementarities between consumption at different periods, the positive correlation does matter: it increases the degree of risk aversion when consumptions at different periods are substitutes and decreases that risk when they are complements. This explains the sign of the correcting term in (11). The magnitude of the correction depends on the number of correlations at play, and it is therefore increasing (in absolute value) with the number of remaining periods of life. This explains the horizon effect that we obtain.

\section{Are Consumptions at Different Ages Specific Substitutes or Specific Complements?}

Equation (11) shows that the relation between relative risk aversion and horizon length depends on the sign of the coefficients of specific substitutability. It would thus appear important to see if there are any empirical findings or theoretical arguments that suggest a particular sign for the $\kappa_{i j}$.

To our knowledge, the only ${ }^{4}$ paper to provide estimates of cross "want elasticities" is Browning (1991). For reasons of parsimony, Browning considers such elasticities to be nonzero only for expenditures in adjacent time periods (expenditures at date $t$ interact only with expenditures at dates

\footnotetext{
${ }^{4}$ There is, however, an empirical literature on the estimation of Frisch intertemporal demand functions initiated by the influential study of labor supply by MaCurdy (1981).
} 
$t-1, t$, and $t+1)$. He finds that such interactions are small but nonnegligible. Most types of expenditures seem to be want independent, but expenditures on durables are found (as expected) to be specific substitutes with themselves in adjacent periods. ${ }^{5}$

Hayashi (1985) also provides some support for the presumption that consumptions at different moments in time are substitutes. Although Hayashi does not estimate "want elasticities", his findings indicate that changes in consumption are strongly negatively autocorrelated. Hayashi attributes such a result to the "durability of consumption". This is actually another way of expressing that consumptions at different moments in time are substitutes.

Theoretical arguments can also be given for why consumptions at different dates can be specific substitutes. This involves the notion of "temporal risk aversion" or "intertemporal correlation aversion" introduced by Richard (1975). To illustrate this notion, consider for example two dates $(n=1,2)$ and two intertemporal lotteries:

$$
L_{1}=\left\{\begin{array}{l}
\left(C_{1}, C_{2}\right) \\
\left(c_{1}, c_{2}\right)
\end{array} \quad \text { and } \quad L_{2}=\left\{\begin{array}{l}
\left(C_{1}, c_{2}\right) \\
\left(c_{1}, C_{2}\right)
\end{array}\right.\right.
$$

where each outcome (in both lotteries) occurs with equal probability. Assume that $c_{1}<C_{1}$ and $c_{2}<C_{2}$. An individual with additively separable preferences (i.e., $\left.U\left(C_{1}, C_{2}\right)=U_{1}\left(C_{1}\right)+U_{2}\left(C_{2}\right)\right)$ is indifferent between

\footnotetext{
${ }^{5}$ Browning also finds that fuel is a specific complement with itself, but the coefficient is smaller.
} 
$L_{1}$ and $L_{2}$, since both lotteries give the same sum of expected utilities $(1 / 2)\left(U_{1}\left(c_{1}\right)+U_{1}\left(C_{1}\right)\right)+(1 / 2)\left(U_{2}\left(c_{2}\right)+U_{2}\left(C_{2}\right)\right)$. But this is generally not the case when preferences are not additively separable. Now we say that an individual is "averse to intertemporal correlation" if he prefers $L_{2}$ to $L_{1}$. Intuitively, she prefers having some of the worst and some of the best to taking a chance on all of the worst or all of the best. Such a pattern happens when $\partial^{2} U / \partial C_{1} \partial C_{2}<0$ and hence when goods 1 and 2 are specific substitutes.

In the $N$-period model, it is no longer true that aversion to intertemporal correlation and positive specific substitutability are equivalent properties. However, the equivalency holds locally when we consider weak interactions. Indeed, from Lemma 3 (see Appendix) we know that, when interactions are weak, we have

$$
\left[D^{2} U\right]_{i j}^{-1} \approx-\frac{\frac{\partial^{2} U}{\partial C_{i} \partial C_{j}}}{\frac{\partial^{2} U}{\left(\partial C_{i}\right)^{2}} \frac{\partial^{2} U}{\left(\partial C_{j}\right)^{2}}} .
$$

Thus, when interactions are weak, preferences that exhibit aversion to intertemporal correlation also exhibit positive specific substitutability.

Another point can be made by comparing relative risk aversion and intertemporal elasticity of substitution. It is well known that the standard specification of the life-cycle model with additive preferences and isoelastic instantaneous utility functions implies that relative risk aversion equals the inverse of intertemporal elasticity of substitution. The most popular way to break this unpleasant relation between two apparently distinct concepts 
is to relax the von Neumann-Morgenstern axioms and follow the theory developed by Kreps and Porteus (1978) on temporal lotteries (see e.g. Epstein and Zin 1989; Farmer 1990; Weil 1990; Campbell 1993). However the relation can also be broken while remaining within the standard von Neumann-Morgenstern framework on atemporal lotteries. Actually, within this framework the (local) relative risk aversion always equals the inverse of the (local) intertemporal elasticity of substitution if and only if the utility function is additively separable (see Bommier 2003). Moreover, the difference between (local) relative risk aversion and (local) intertemporal elasticity of substitution is precisely determined by aversion to intertemporal correlation. Because aversion to intertemporal correlation and specific substitutability are closely related (at least when interactions are weak), the coefficients of specific substitutability considered in this paper can be related to the difference between local measures of intertemporal elasticity of substitution and local indices of relative risk aversion. By definition, the elasticity of substitution between consumptions at dates $i$ and $j$ (holding consumption in other periods constant) is given by ${ }^{6}$

$$
\sigma_{i j}=\frac{\left(C_{i} \frac{\partial U}{\partial c_{i}}\right)^{-1}+\left(C_{j} \frac{\partial U}{\partial c_{j}}\right)^{-1}}{-\frac{\partial^{2} U /\left(\partial C_{i}\right)^{2}}{\left(\partial U / \partial C_{i}\right)^{2}}+2 \frac{\partial^{2} U / \partial C_{i} \partial C_{j}}{\left(\partial U / \partial C_{i}\right)\left(\partial U / \partial C_{j}\right)}-\frac{\partial^{2} U /\left(\partial C_{j}\right)^{2}}{\left(\partial U / \partial C_{j}\right)^{2}}} .
$$

It follows easily from (10) and (13) that, to a first-order approximation, the

\footnotetext{
${ }^{6}$ Note that, with additively separable preferences and an isoelastic instantaneous utility function, the $\sigma_{i j}$ equal a constant - the "intertemporal elasticity of substitution".
} 
coefficients of specific substitutability are also given by

$$
\kappa_{i j} \approx \frac{1}{2 r_{i} r_{j}}\left[\frac{\alpha_{j}}{\alpha_{i}+\alpha_{j}}\left(r_{i}-\frac{1}{\sigma_{i j}}\right)+\frac{\alpha_{i}}{\alpha_{i}+\alpha_{j}}\left(r_{j}-\frac{1}{\sigma_{i j}}\right)\right]
$$

where

$$
r_{i}=-\frac{C_{i} \frac{\partial^{2} U}{\left(\partial C_{i}\right)^{2}}}{\frac{\partial U}{\partial C_{i}}}
$$

is the relative risk aversion index with respect to consumption in period $i$ and $\alpha_{i}$ is the budget share spent in period $i$. Thus, if all local coefficients of relative risk aversion are greater than the inverse of intertemporal elasticity of substitution between any two periods, then preferences exhibit positive specific substitutability. Empirical measures of local relative risk aversion indices and intertemporal elasticities of substitution could then be used to determine whether consumptions at different dates are specific complements or rather specific substitutes — and ultimately to elucidate the relation between horizon length and intertemporal risk aversion. ${ }^{7}$ Unfortunately, both

\footnotetext{
${ }^{7}$ Equation (11) can be rewritten as
}

$$
T_{n}\left(C^{*}\right)=\sum_{i \geq n} \alpha_{i}^{n} t_{i}\left(C^{*}\right)-\sum_{\substack{i, j \geq n \\ i \neq j}} \frac{\alpha_{j}^{n}\left(r_{i}-1 / \sigma_{i j}\right)}{r_{i} r_{j}}+\kappa o(\kappa) .
$$

It is therefore clear that the correcting term that appears in (11) and accounts for interactions between consumption at different dates can also be written, to a first-order approximation, as a weighted sum of differences between local relative risk aversion indices and the inverse of local intertemporal elasticities of substitution. In particular, when all local relative risk aversion indices are greater than inverse elasticities of substitution, the correction is negative. Moreover, for the same reasons as given after (12), the magnitude of the correction typically increases with horizon length. 
risk aversion and intertemporal elasticities of substitution are particularly difficult to measure, and the empirical literature remains inconclusive about the sign and the magnitude of these differences.

To conclude this section on intertemporal interactions, we discuss the relation between specific interactions and habit formation. For technical reasons, most papers on habit formation assume that the intertemporal utility function has some form of additive structure. Namely, the $N$-period utility function is generally specified as the sum of instantaneous utility functions:

$$
U^{H}\left(C_{1}, \ldots, C_{N}\right)=\sum_{i=1}^{N} V_{i}\left(C_{i}, X_{i}\right),
$$

where $X_{i}$ (the stock of habits in period $i$ ) is positively related to consumptions in the previous periods. The cross-derivatives $\partial^{2} V_{i} / \partial C_{i} \partial X_{i}$ are assumed to be positive. Of course, since $X_{i}$ depends on consumption in previous periods, such preferences are not additively separable. Still, the structure of (14) imposes strong restrictions. In particular, since $\partial X_{i} / \partial C_{j}>0$ for any $j<i$ and since $\partial^{2} V_{i} / \partial C_{i} \partial X_{i}>0$ for any $i$, we have $\partial^{2} U^{H} / \partial C_{i} \partial C_{j}>0$ for any $i \neq j$. Hence, preferences as represented by (14) exhibit negative intertemporal correlation aversion, and the coefficients of specific substitutability are therefore negative. Thus, it appears that most papers on habit formation do assume that consumptions at different dates are specific complements, which implies that there is a positive relationship between risk tolerance and horizon length. However, this follows only because these papers rely on the specific structure of (14). For a general (not additively 
separable) specification, there is no systematic relation between habit formation and specific substitutability. Habit formation (as defined in Becker and Murphy 1988) is equivalent to the notion of adjacent complementarity introduced by Ryder and Heal (1973). Preferences are said to exhibit adjacent complementarity if

$$
\frac{\partial}{\partial C_{i}}\left(\frac{\frac{\partial U}{\partial C_{i+1}}}{\frac{\partial U}{\partial C_{i+2}}}\right)>0 \text { forall } i \leq N-2
$$

(i.e., if marginal rate of substitution between present and future consumption increases with past consumption). This is an ordinal notion that is preserved under any increasing transformation. In other words, if a utility function $U$ exhibits adjacent complementarity then any monotonic transformation $\widetilde{U}=f(U)$ will also exhibit adjacent complementarity. However, for $f$ sufficiently concave, consumptions at different dates become specific substitutes. Preferences involving habit formation can therefore exhibit specific complementarity as well as specific substitutability.

\section{Concluding Remarks}

We have shown in this paper that interactions between consumptions at different dates could generate variations of relative risk aversion during the life cycle, even if tastes do not vary with age and even if we control for wealth effects. More specifically, Proposition 2 has shown that, if the von Neumann-Morgenstern utility of an individual is a concave transformation 
of an additively separable function, then relative risk aversion decreases with age along any stationary consumption path. Proposition 3 extends this result to a more general form of interactions and to nonstationary consumption paths, and this enables us to evaluate the bias introduced by the additive separability assumption in the estimation of intertemporal risk aversion. This bias is approximately equal to minus the sum of specific substitutability coefficients as weighted by budget shares. The bias is typically larger (in absolute values) for young individuals — who still have many periods to live- than for older ones.

Our results can be used in different ways. We can apply them to models that assume simple specifications for the utility function. Take, for example, an exponential transformation of a sum of CRRA utilities:

$$
U(C)=-\frac{1}{k} \exp \left\{-k \sum_{i=1}^{N} \frac{C_{i}^{1-\gamma}-1}{1-\gamma}\right\}
$$

where $k$ is positive. A simple application of equation (9) immediately yields the relative risk aversion coefficient $R_{n}$ of an individual of age $n$ along any constant consumption path:

$$
R_{n}=\frac{1}{T_{n}}=\gamma+k(N-n+1) C^{1-\gamma}
$$

As expected, $R_{n}$ decreases with $n$ because $k>0$.

The utility function that we used for our illustrative example in Section 3 is obtained for $\gamma=1$ and $N=2$. From (16) we obtain $T_{1}=1 /(1+2 k)$ and $T_{2}=1 /(1+k)$, which (when combined with Lemma 2 ), leads to the 
result obtained in Section 3; see (5). We have thus found two ways to obtain the same results. The first way, which we followed in Section 3, consists of providing an explicit solution to the portfolio choice problem and deriving some of its properties; this is indisputably the most popular approach in the finance literature. The second way consists of looking at marginal properties of the utility function and in particular at our measure of intertemporal risk tolerance indices. The first method has an obvious advantage: it works even when the share of risky assets is relatively large and portfolio risks are not small. But it has also a major drawback: it can only work when it is possible to derive a closed-form solution to the portfolio choice problem. Needless to say, the number of specifications for which such closed-form solutions are available is extremely limited. The literature has naturally focused on these particular specifications, but there is no reason to suppose they should fit observed behavior particularly well.

The alternate route that we have followed in this paper does not suffer from such technical constraints. In fact, our results make it possible to derive estimates of how risk aversion varies with age - even if we only have only a limited and local knowledge of individual preferences. Consider, for example, equation (11):

$$
T_{n}\left(C^{*}\right)=\sum_{i \geq n} \alpha_{i}^{n} t_{i}\left(C^{*}\right)-\sum_{\substack{i, j \geq n \\ i \neq j}}\left(\alpha_{i}^{n}+\alpha_{j}^{n}\right) \kappa_{i j}+\kappa o(\kappa) .
$$

Budget shares $\alpha_{i}^{n}$ usually are relatively well observed. The other ingredients 
needed to obtain intertemporal risk tolerance indices as a function of age are local estimates of the instantaneous risk tolerance indices, $t_{i}\left(C^{*}\right)$, and the coefficients of specific substitutability, $\kappa_{i j}$. Imagine for instance that all the budget shares are observed to be equal. Also assume that, at the optimal consumption path, there is no variation in the instantaneous indices of relative risk tolerance during the life cycle $\left(t_{i}\left(C^{*}\right)=1 / \gamma\right)$ and that the coefficients of specific substitutability are of the form $\kappa_{i j}=\kappa \rho^{|i-j|-1}$. Then the parameter $\kappa$ gives the strength of the interactions while $\rho$ determines their shape (specific substitution decreases with time distance if $\rho<1$ and increases with time distance if $\rho>1$ ). In such a case, (11) leads to

$$
\begin{aligned}
T_{n}\left(C^{*}\right) & \approx \frac{1}{\gamma}-\frac{2 \kappa}{N-n+1} \sum_{\substack{i, j \geq n \\
i \neq j}} \rho^{|i-j|-1} \\
& =\frac{1}{\gamma}-\frac{4 \kappa}{N-n+1}\left[\frac{\rho\left(\rho^{N-n}-1\right)+(N-n)(1-\rho)}{(1-\rho)^{2}}\right]
\end{aligned}
$$

This relation between relative risk tolerance and horizon length is shown in Figure 1; the corresponding picture for the relative risk aversion is displayed in Figure 2. In particular, we observe that the relation between relative risk tolerance and horizon length is convex (concave) if specific substitutability between consumption levels at different dates decreases (increases) with time distance between these dates.

An unresolved issue is whether considering risk aversion suffices to provide a relatively good approximation of the life-cycle financial strategy of individuals. We have shown that this is the case when the share of risky 
assets are small, but one may wonder whether it remains true when agents take noninfinitesimal risks. In this case, an individual's wealth follows a random path, and the dynamic aspects of the problem (as emphasized in Gollier and Zeckhauser 2002) must be considered. In particular, it matters whether risk tolerance indices are convex or concave with respect to wealth. Whether these considerations are likely to generate larger effects than those discussed in this paper is difficult to tell. However, one can reasonably presume that the fundamental properties of preferences that drive the result of Gollier and Zeckhauser (and are related to the fourth derivative of the utility function) will be more difficult to test empirically than the complementarities and substitutabilities that we have discussed here, which depend on second derivatives only. In particular, the impact of nonseparability analyzed in this paper is already present when instantaneous preferences are CRRA, whereas the phenomenon studied by Gollier and Zeckhauser would vanish in this case. 


\section{$9 \quad$ Appendix}

Proof of Lemma 1. We have

$$
\theta^{*}(k)=\arg \max \{\varphi(\theta, k)\}
$$

where

$$
\varphi(\theta, k)=\frac{1-E[1+\theta \tilde{R}]^{-k}}{k}
$$

To establish that $\theta^{*}(\cdot)$ is decreasing, it is enough to show that $\left(\partial^{2} \varphi / \partial \theta \partial k\right)\left(\theta^{*}(k), k\right)<$

0 (single crossing property).

Indeed,

$$
\frac{\partial \varphi}{\partial \theta}=E\left[\tilde{R}(1+\theta \tilde{R})^{-i-1}\right]
$$

and

$$
\frac{\partial^{2} \varphi}{\partial k \partial \theta}=-E\left[\tilde{R} \ln (1+\theta \tilde{R})(1+\theta \tilde{R})^{-k-1}\right]
$$

Now, for all $\theta>0$ and all $\tilde{R}$ we have $\tilde{R} \ln (1+\theta \tilde{R})>0$. Thus $\partial^{2} \varphi / \partial k \partial \theta<$ 0 . That $\theta^{*}(k)>0$ follows from our assumption that $E[\tilde{R}]>0$ (since $(\partial \varphi / \partial \theta)(0, k)=E[\tilde{R}])$.

Proof of Proposition 3. The proof relies on two simple ingredients.

1. A formula due to Hanoch (1977) that relates $T(C)$, the intertemporal risk tolerance index along a consumption path $C$, to the matrix $\left[D^{2} U\right]^{-1}(C)$ and the utility gradient $\nabla U(C)$ :

$$
T(C)=\frac{{ }^{t} \nabla U\left[D^{2} U\right]^{-1} \nabla U}{t_{C \nabla U}}
$$


2. The following linear algebra lemma about the inverse of nonsingular matrices that are almost diagonal.

Lemma 3. Consider a matrix $M=\left[m_{i j}\right]$ with $m_{i i} \neq 0$ for all $i$, and note that $m=\sup _{i \neq j}\left|m_{i j}\right|$. Then, when $m$ is small enough, $M$ is nonsingular and the $(i, j)$ th elements of $M^{-1}$ are given by

$$
\begin{aligned}
{\left[M^{-1}\right]_{i i} } & =\frac{1}{m_{i i}}+m o(m) \quad \text { and } \\
{\left[M^{-1}\right]_{i j} } & =-\frac{m_{j i}}{m_{i i} m_{j j}}+m o(m) \quad \text { if } i \neq j
\end{aligned}
$$

where $o(m) / m \rightarrow 0$ when $m \rightarrow 0$.

Proof. Take $M$ nonsingular, with $m=\sup _{i \neq j}\left|m_{i j}\right|$ close to zero, and define $\varphi_{i j}[M]=\left[M^{-1}\right]_{i j}$, the generic term of $M^{-1}$. The value of $\varphi_{i j}[M]$ is given explicitly by the classical formula

$$
\varphi_{i j}[M]=\frac{(-1)^{i+j} \operatorname{det}\left[M_{j i}\right]}{\operatorname{det}[M]}
$$

where $\operatorname{det}[A]$ denotes the determinant of any square matrix $A$ and $M_{i j}$ is the submatrix obtained by deleting the $i$ th row and the $j$ th column of $M$. Define $\Delta=\operatorname{diag}[\mathrm{M}]$, the matrix obtained from $M$ by deleting off-diagonal terms. Because $\varphi_{i j}$ is differentiable on its domain (we note its derivative by $D \varphi_{i j}$ ), we can write a Taylor expansion around $\Delta$ that is valid for $m$ small:

$$
\varphi_{i j}[M]=\varphi_{i j}[\Delta]+D \varphi_{i j}[\Delta](M-\Delta)+m o(m)
$$

where $o(m) / m \rightarrow 0$ when $m \rightarrow 0$. 
Since $\varphi_{i i}(\Delta)=\left(m_{i i}\right)^{-1}$ and $\varphi_{i j}(\Delta)=0$ for $i \neq j$, Lemma 3 is proven if we can establish that $D \varphi_{i i}(\Delta)(M-\Delta)=0$ and $D \varphi_{i j}(\Delta)(M-\Delta)=$ $(-1)^{i+j} m_{j i} / m_{i i} m_{j j}$ for $i \neq j$. To do so, let us first compute the partial derivatives of $\varphi_{i j}$ by differentiating (A1) with respect to $m_{k l}$ (for arbitrary $k, l)$. This yields

$\frac{\partial \varphi_{i j}}{\partial m_{k l}}(\Delta)=\left.\frac{(-1)^{i+j}}{\operatorname{det}(\Delta)} \frac{\partial\left(\operatorname{det}\left(M_{j i}\right)\right)}{\partial m_{k l}}\right|_{M=\Delta}-\left.(-1)^{i+j} \frac{\operatorname{det}\left(\Delta_{j i}\right)}{\operatorname{det}^{2}(\Delta)} \frac{\partial(\operatorname{det}(M))}{\partial m_{k l}}\right|_{M=\Delta}$.

Now

$$
\begin{aligned}
\left.\frac{\partial\left(\operatorname{det}\left(M_{j i}\right)\right)}{\partial m_{k l}}\right|_{M=\Delta} & =(-1)^{i+j-1} \frac{\operatorname{det}(\Delta)}{m_{i i} m_{j j}} \text { if } k=i \text { and } l=j, \\
& =0 \text { otherwise; }
\end{aligned}
$$

and

$$
\begin{aligned}
\frac{\partial[\operatorname{det}[M]]}{\partial m_{k l}} & =\frac{\operatorname{det}([\Delta]}{m_{k k}} \text { if } k=l \\
& =0 \quad \text { otherwise. }
\end{aligned}
$$

Since $(M-\Delta)_{k l}=m_{k l}$ if $k \neq l$ and zero otherwise, if follows that

$$
\begin{aligned}
D \varphi_{i j}(\Delta)(M-\Delta) & =\frac{-n_{i j}}{m_{i i} m_{j j}} \text { if } i \neq j \\
& =0 \quad \text { if } i=j .
\end{aligned}
$$

Thus we have established the desired result:

$$
\begin{aligned}
D \varphi_{i j}(\Delta)(M-\Delta) & =0 \text { if } i=j, \\
& =-\frac{m_{j i}}{m_{i i} m_{j j}} \text { if } i \neq j .
\end{aligned}
$$


Proof of Proposition 3. We are now in a position to prove Proposition 3.

For any past consumption $\left(C_{1}^{*}, \ldots, C_{n-1}^{*}\right)$, we define

$$
U_{n}\left(C_{n}, \ldots, C_{N}\right)=U\left(C_{1}^{*}, \ldots, C_{n-1}^{*}, C_{n}, \ldots, C_{N}\right),
$$

Using Hanoch's formula (see Hanoch 1977, p. 416) in developed form yields

$$
T_{n}(C)=-\sum_{i=n}^{N} \frac{\left[D^{2} U_{n}\right]_{i i}^{-1} u_{i}^{2}}{{ }^{t} c \nabla U_{n}}-\sum_{\substack{i \neq j \\ n \leq i, j \leq N}} \frac{\left[D^{2} U_{n}\right]_{i j}^{-1} u_{i} u_{j}}{{ }^{t} c \nabla U_{n}},
$$

where $u_{i}=\partial U_{n} / \partial C_{i}=\partial U / \partial C_{i}$. Recall the expressions of the relative budget shares $\alpha_{i}^{n}=C_{i} u_{i} /{ }^{t} c \nabla U_{n}$, specific substitutability coefficients $\kappa_{i j}=$ $\left[D^{2} U\right]_{i j}^{-1} u_{i} u_{j} / c_{i} u_{i}+c_{j} u_{j}$, and instantaneous risk tolerance coefficients $t_{i}=$ $-u_{i} / C_{i}\left(\partial^{2} U / \partial C_{j}^{2}\right)$. Lemma 3 shows that, when $\kappa=\max _{i \neq j}\left|\kappa_{i j}\right|$ is small,

$$
\left[D^{2} U_{n}\right]_{i i}^{-1}=\left(\frac{\partial^{2} U_{n}}{\partial C_{i}^{2}}\right)^{-1}+\kappa o(\kappa)=\left(\frac{\partial^{2} U}{\partial C_{i}^{2}}\right)^{-1}+\kappa o(\kappa)
$$

and thus

$$
-\frac{\left[D^{2} U_{n}\right]_{i i}^{-1} u_{i}^{2}}{{ }^{t} c \nabla U_{n}}=\alpha_{i}^{n} t_{i}+\kappa o(\kappa) .
$$

Moreover, for $i \neq j$ we have $\left[D^{2} U_{n}\right]_{i j}^{-1}=\left[D^{2} U\right]_{i j}^{-1}+\kappa o(\kappa)$ by Lemma 3 ,

so

$$
\frac{\left[D^{2} U_{n}\right]_{i j}^{-1} u_{i} u_{j}}{{ }^{t} c \nabla U_{n}}=\left(\alpha_{i}^{n}+\alpha_{j}^{n}\right) \kappa_{i j}+\kappa o(\kappa) .
$$

Therefore, (A2) can be written as

$$
T_{n}(C)=\sum_{i=n}^{N} \alpha_{i}^{n} t_{i}-\sum_{\substack{i \neq j \\ n \leq i, j \leq N}}\left(\alpha_{i}^{n}+\alpha_{j}^{n}\right) \kappa_{i j}+\kappa o(\kappa),
$$

and the proof of Proposition 3 is complete. 


\section{References}

Ahn, Chang M. (1989) "The Effect of Temporal Risk Aversion on Optimal Consumption, the Equity Premium, and the Equilibrium Interest Rate." Journal of Finance, 44, 1411-1420.

Ameriks, John and Stephen Zeldes (2001). "How Do Household Portfolio

Shares Vary with Age?" Working Paper no. 6-120101, TIAA-CREF Institute.

Arrow, Kenneth J. (1971). Essays in the Theory of Risk Bearing. Markham. Barsky, Robert B., Thomas F. Juster, Miles S. Kimball and Matthew D. Shapiro, 1997, "Preference Parameters and Behavioral Heterogeneity: An Experimental Approach in the Health and Retirement Study." Quarterly Journal of Economics, 112, 537-579.

Becker, Garry and Kevin Murphy (1988). "A Theory of Rational Addiction." Journal of Political Economy, 96, 675-700.

Bodie, Zvi, Robert C. Merton, and William F. Samuelson (1992) "Labor Supply Flexibility and Portfolio Choice in a Life-Cycle Model.” Journal of Economic Dynamics and Control, 16, 427-449.

Bommier, Antoine (2003). "Risk Aversion, Intertemporal Elasticity of Substitution and Correlation Aversion." Working Paper no 03-07, LEA-INRA. Browning, Martin (1991). "A Simple Nonadditive Preference Structure for Models of Household Behavior over Time." Journal of Political Economy, Vol. 99, 607-637. 
Campbell, John. (1993). "Intertemporal Asset Pricing without Consumption Data." American Economic Review, 83, 487-512.

Carrasco, Raquel, Jos Labeaga and David López-Salido (2005). "Consumption and Habits: Evidence from Panel Data." Economic Journal, 115, 144165.

Constantinides, George M. (1990). "Habit Formation: A Resolution of the Equity Premium Puzzle." Journal of Political Economy, 98, 519-543.

Dynan, Karen (2000). "Habit Formation in Consumer Preferences: Evidence from Panel Data." American Economic Review, 90(3), 391-406.

Epstein, Larry G. and Stanley E. Zin (1989). "Substitution, Risk Aversion, and the Temporal Behavior of Consumption and Asset Returns: A Theoretical Framework." Econometrica, 57, 937-69.

Farmer, Roger (1990). "RINCE Preferences". Quarterly Journal of Economics, 105, 43-60.

Frisch, Ragnar (1959). “A Complete Scheme for Computing All Direct and Cross Demand Elasticities in a Model with Many Sectors." Econometrica, $27,177-196$.

Gollier, Christian and Richard Zeckhauser (2002) “Time Horizon and Portfolio Risk." Journal of Risk and Uncertainty, 49, 195-212.

Gorman, William M. (1968) "The Structure of Utility Functions." Review of Economic Studies, 35, 367-390.

Guiso, Luigi, Haliassos, Michael, and Tullio Jappelli (2002a). Household 
Portfolios. MIT Press.

Guiso, Luigi, Haliassos, Michael, and Tullio Jappelli (2002b). Stockholding in Europe. Palgrave Macmillan.

Guiso, Luigi and Paiella, Monica (2001). Risk Aversion, Wealth and Background Risk. CEPR Discussion Paper no. 2728. London, Centre for Economic Policy Research. http://www.cepr.org/pubs/dps/DP2728.asp.

Hanoch, Giora (1977). "Risk Aversion and Consumer Preferences." Econometrica, 45, 413-426.

Hayashi, Fumio (1985). "The Effect of Liquidity Constraints on Consumption: A Cross-Sectional Analysis." Quarterly Journal of Economics, 100, $183-206$.

Houthakker, Hendrick S. (1960). “Additive Preferences.” Econometrica, 28, $244-257$.

Karni, Edi (1979). "On Multivariate Risk Aversion." Econometrica, 47, $1391-1401$.

Karni, Edi (1983). "On the Correspondence between Multivariate Risk Aversion and Risk Aversion with State-Dependent Preferences." Journal of Economic Theory, 30, 230-242.

Kihlstrom, Richard, and Leonard Mirman (1974). "Risk Aversion with Many Commodities." Journal of Economic Theory, 8, 361-88.

Kreps, David and Evan Porteus (1978). "Temporal Resolution of Uncertainty and Dynamic Choice Theory," Econometrica, 46(1), 185-200. 
MaCurdy, Thomas (1981). "An Empirical Model of Labor Supply in a LifeCycle Setting." Journal of Political Economy, 89, 1059-1085.

Merton, Robert (1969). "Lifetime Portfolio Selection under Uncertainty: The Continuous-Time Case." Review of Economics and Statistics, 51, 247257.

Muellbauer, John (1988). "Habits, Rationality and Myopia in the Life-cycle Consumption Function." Annales d'Economie et de Statistique, 9, 47-70. Pratt, John (1964). "Risk Aversion in the Small and in the Large," Econometrica, 32, 122-136.

Pye, Gordon (1973). "Lifetime Portfolio Selection in Continuous Time for a Multiplicative Class of Utility Functions." The American Economic Review, $63(5), 1013-1016$.

Richard, Scott (1975). "Multivariate Risk Aversion, Utility Independence and Separable Utility Functions." Management Science, 22, 12-21.

Ryder, Harl and Geoffrey Heal (1973). "Optimal Growth with Intertemporally Dependent Preferences." Review of Economic Studies, 40, 1-31. Samuelson, Paul A. (1969). "Lifetime Portfolio Selection by Dynamic Stochastic Programming." Review of Economics and Statistics, 51, 239-246. Samuelson, Paul A. (1989). "The Judgment of Economic Science On Rational Portfolio Management: Indexing, Timing, and Long-Horizon Effects." Journal of Portfolio Management, Fall, 4-12.

Weil, Philippe (1990). “Nonexpected Utility in Macroeconomics." Quarterly 
Journal of Economics, 105, 29-42. 